\title{
Early Cerebrovascular Silent Changes in Long-Standing End-Stage Renal Disease Patients on Hemodialysis Value of Adding Advanced Unenhanced MRI Sequences to Imaging Protocols
}

\author{
Marwa Hassan Madboly, Mohamed Fathy Khater, Hosam N. Almassry, Fatma Zaiton, Ali M. Hassanin \\ Department of Radiodiagnosis, Faculty of Medicine, Zagazig University, Egypt \\ Corresponding author: Marwa Hassan Madboly, Mobile: (+20) 01017219552, E-Mail: abdelshafi.marwa@ yahoo.com
}

\begin{abstract}
Background: End-Stage Renal Disease (ESRD) patients on hemodialysis suffered from many central and peripheral neurological insults. Aim of the work:This study aimed to assess the value of using MRA, MRV, and SWAN sequences in early visualization of the silent cerebrovascular complications in those patients.

Patients and Methods: Our study was conducted on forty-five patients with well-documented ESRD on regular hemodialysis for more than 5 years with no neurological manifestation, all undergone unenhanced MRI, DWI with ADC and measuring the ADC value, SWAN, MRA, and MRV.

Results: we found that $11 \%$ of cases have acute infarction at the basal ganglia region. $36.7 \%$ of patients are diagnosed with intracerebral hemorrhage, only $26.7 \%$ of them are seen by the conventional MRI, and $16.7 \%$ of cases showed microbleeds on SWAN with normal conventional MRI. The sensitivity and specificity of SWAN with ADC value to detect brain hemorrhage are $100 \%$ and $88.8 \%$ respectively.

Conclusion: Visualization of cerebrovascular complications such as infarction, hemorrhage, atherosclerosis, and arterial and venous occlusion using DWI with ADC, MRA, MRV, and SWAN and is very useful in early management and better prognosis of ESRD patients even with silent complications that don't give up symptoms with high sensitivity and specificity of SWAN in early detection of hemorrhage and microbleeds.
\end{abstract}

Keywords: Cerebrovascular, Hemodialysis, DWI, MRI.

\section{INTRODUCTION}

Uremic encephalopathy (UE) is a metabolic disease occurring in patients with end-stage renal disease (ESRD) and resulting from the accumulation of uremic toxins such as creatinine and BUN, it may have acute or subacute onset of reversible neurological symptoms ${ }^{(\mathbf{1})}$. These neurologic complications can affect negatively the ESRD prognosis. Early diagnosis of these complications may carry the hope for optimum care and re-evaluation of treatment strategic plans for ESRD patients ${ }^{(2)}$. Cerebrovascular complications of ESRD patients are infarction, hemorrhage ${ }^{(3)}$, venous, arterial occlusion, atherosclerosis, and posterior reversible encephalopathy syndrome PRES ${ }^{(4)}$.

The white matter and basal ganglia are the most affected areas as well as the cerebral cortices ${ }^{(5)}$. The vascular complications are detected easily by using MRI even without the presence of neurovascular symptoms and using MRI series like diffusion-weighted imaging DWI with apparent diffusion coefficient ADC, magnetic resonance angiography MRA, magnetic resonance venography MRV, and susceptibility-weighted Angiography SWAN help in the detection of some brain insults that can't be visualized on the usual conventional MRI ${ }^{\left({ }^{6}\right)}$.In this study, we aim to early detection of silent cerebrovascular changes of ESRD patients using a new MRI series adding to the value of the usual conventional MRI.

\section{PATIENTS AND METHODS}

Our study included 45 patients with end-stage renal disease on regular hemodialysis, conducted at the
Department of Radiodiagnosis, Faculty of Medicine, Zagazig University, during the period from July 2019 to April 2021.

Inclusion criteria were (1) ESRD patients of both genders on regular hemodialysis more than 5 years, (2) age group 20-40 years. Patients with the following criteria were excluded (1) history of any neurological disease, diabetes, and hypertension (2) patients below 20 years or exceeding 40 years, and (3) Contraindication to MRI like pacemaker, cochlear implant.

\section{Ethical considerations}

This prospective cross-sectional study was conducted after approved by the Ethics Board of Zagazig University.Informed consent was obtained from all patients before the study. We followed the ethical principles of the Declaration of Helsinki during the preparation of this study.

MRI examination: MR imaging was performed using 1.5 Telsa MR Scanner (GE MR Signa Medical system) using the head coil.

MR Protocol (Scan protocol and parameters):

The examination protocol started with Conventional Magnetic Resonance Imaging, at first Sagittal T1WI used as a localizer $(\mathrm{TE}=10-15 \mathrm{~m} / \mathrm{sec} \& \mathrm{TR}=400-600$ $\mathrm{m} / \mathrm{sec}$ ), axial spin echo sequences, short TR/TE (T1Weighted Images): (TE $=10-15 \mathrm{M} / \mathrm{sec}, \mathrm{TR}=400-600$ $\mathrm{m} / \mathrm{sec}$ ), Axial and coronal fast spin echo, long TR/TE (T2- Weighted Images): (TE=70-110 m/sec, TR=2400$3500 \mathrm{~m} / \mathrm{sec}$ ) and Axial Fluid.

Attenuated Inversion-Recovery sequences (FLAIR): $(\mathrm{TE}=119-150 \mathrm{~m} / \mathrm{sec}, \mathrm{TI}=2470-2800 \mathrm{~m} / \mathrm{sec}, \mathrm{TR}=6000$ - 
$6400 \mathrm{~m} / \mathrm{sec}$ ). The FOV= $24-18 \mathrm{~cm}$ in axial images and $30-22 \mathrm{~cm}$ in coronal images, Matrix (frequency x phase) $192 \times 160$, Slice thickness $=6 \mathrm{~mm}$ with $2 \mathrm{~mm}$ interval (in all sequences).

DWI and ADC with diffusion sensitivities of bvalues $=0$ and $1000 \mathrm{~s} / \mathrm{mm}^{2}$. Sections of $5 \mathrm{~mm}$ thickness, interslice gap of $1 \mathrm{~mm}$, FOV $240 \mathrm{~mm}$, and a matrix of $128 \times 256$ was used for all images.

MRA and MRV; three-dimensional time of flight (3D TOF) MRA was performed for the vertebrobasilar system including the posterior cerebral arteries, the intracranial internal carotid system, including the middle cerebral and the anterior cerebral arteries, at the level of the circle of Willis, with settings of TR35, TE 7.2, Flip angle 20, images interpolated to $1.5-\mathrm{mm}$ slice thickness, matrix $200 / 512$, the field of view 20, scan time $6.18 \mathrm{~min}$ and superior saturation band. MRA images were processed with a maximum intensity projection 3dimensional time-of-flight technique. A review of the source images with the post-processing images MIP was done to overcome any overestimation of the degree of stenosis or any possible artifacts from MIP images. The structures considered for the study include intra-cranial venous structures (superficial, deep venous systems, and dural venous sinuses). Both MRA \& MRV were taken in axial, coronal, and sagittal planes.

SWAN; the susceptibility effect is most visible in non-refocused GE techniques using long echo times (TE), short flip angles, and high field strengths. In SWAN, the images are further processed with a minimum intensity projection algorithm (minIP) to obtain 3-10 mm thick high signal to noise minIP slabs. These minIP images thereby reveal the continuity of tortuous veins across the slices while attenuating the signal coming from the brain tissue. On magnitude images, the longer TE of the SWI sequence (e.g., $40 \mathrm{~ms}$ at 1.5 tesla) allows for more phase dispersion and $\mathrm{T} 2$ shortening of the protons in the local inhomogeneous magnetic field. A low flip angle can keep the CSF brighter than the surrounding parenchyma. Consequently, the magnitude image highlights areas with short $\mathrm{T}_{2} *$ and leads to lower signals in major veins due to the presence of deoxyhemoglobin.

\section{Statistical analysis}

The collected data were coded, processed, and analyzed using the SPSS (Statistical Package for Social Sciences) version 24 for Windows ${ }^{\circledR}$ (IBM SPSS Inc, Chicago, IL, USA). Data were tested for normal distribution using the Shapiro Walk test. Qualitative data were represented as frequencies and relative percentages. Chi-square test $(\chi 2)$ to calculate the difference between two or more groups of qualitative variables. Quantitative data were expressed as mean \pm SD (Standard deviation). Independent samples t-test was used to compare between two independent groups of normally distributed variables (parametric data). Qualitative data were represented as frequencies and percentages. For quantitative variables mean, standard deviation (SD), and (minimum-maximum) were computed. Sensitivity, specificity, predictive value for positive (PVP), predictive value for negative ( $\mathrm{PVN})$, and accuracy were calculated at $95 \% \mathrm{CI}$ to measure the validity. $\mathrm{P}$-value $<0.05$ was considered significant.

\section{RESULTS}

Demographic and clinical data of studied groups: Forty-five patients with ESRD were included, they were 18 female (40\%) and 27 male $(60 \%)$.

MRI data presentation: The most common finding on MRI was old infarctions (70 \%) and cerebral atrophy (60\%), followed by small vessel disease (SVD) (30\%) and sinus venous thrombosis (SVT) $(30 \%)$.

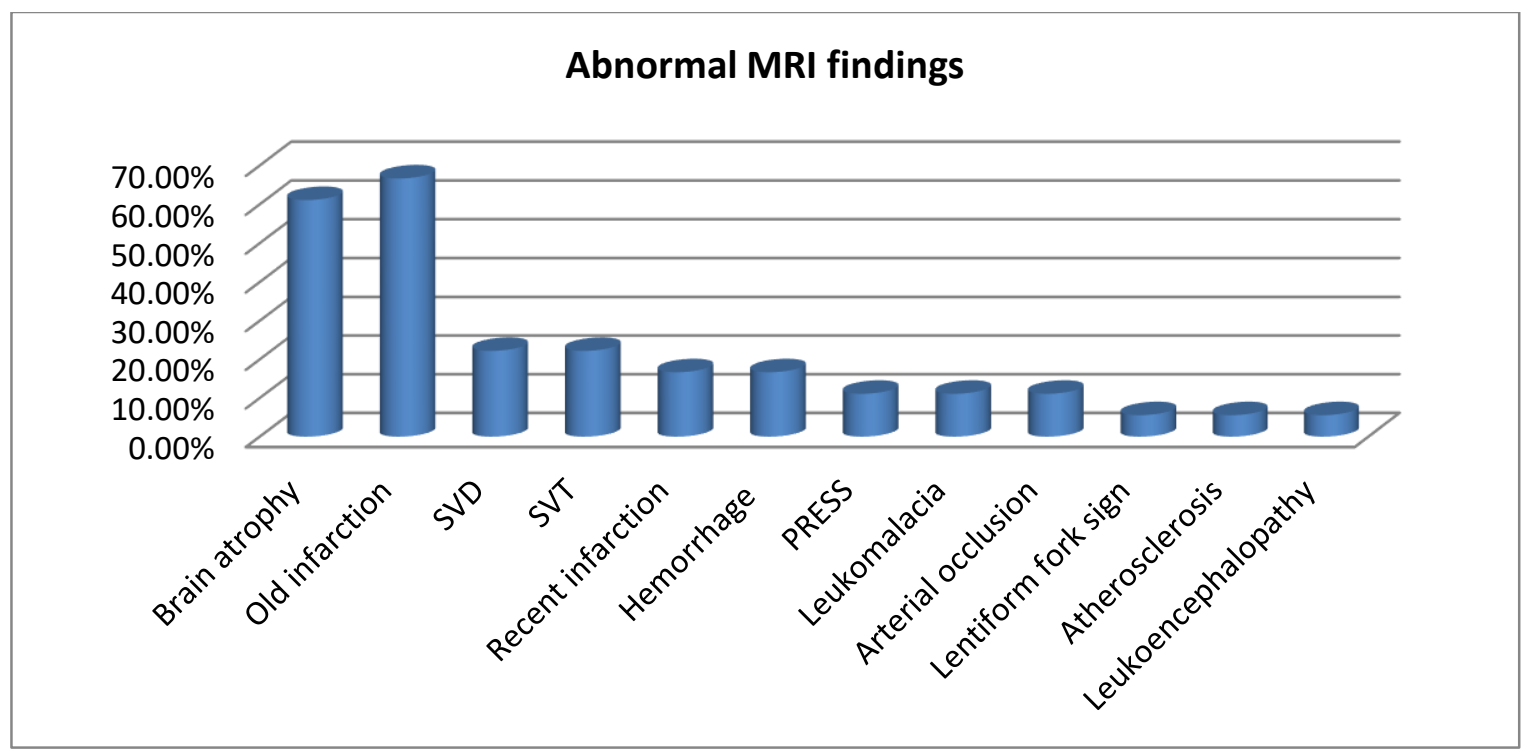

Figure (1): Distribution of different abnormal brain MRI findings (\%) among the cases.

Distribution of patients with chronic infarction in different brain regions noticed more in the white matter (18.8\%) and basal ganglia (18.8\%) while the acute infarction is seen by DWI and ADC showing restriction in the basal ganglia mainly at thalami $(6.6 \%)$ and internal capsule $(4.4 \%)$. 
Table (1): Distribution sites of old and recent infarction of the total population using the conventional MRI and DWI with ADC by percentage (\%).

\begin{tabular}{|l|c|c|c|c|}
\hline & \multicolumn{2}{|c|}{ Old Infarction } & \multicolumn{2}{c|}{ Recent Infarction } \\
\hline \multicolumn{1}{|c|}{ Site of lesion } & Number & Percentage (\%) & Number & Percentage (\%) \\
\hline Multiple WM & 8 & $17.78 \%$ & - & - \\
\hline Thalamic & - & - & 3 & $6.6 \%$ \\
\hline Internal capsule & 1 & $3.3 \%$ & 2 & $4.4 \%$ \\
\hline Cortical & 6 & $14 \%$ & - & - \\
\hline Periventricular & 4 & $8.9 \%$ & - & - \\
\hline Basal ganglia & 8 & $18.8 \%$ & - & - \\
\hline Pons & 3 & $6.7 \%$ & - & - \\
\hline cerebellar & 6 & $13.3 \%$ & - & - \\
\hline
\end{tabular}

Among all patients of the study, the MRV showed about $11.1 \%$ had left transverse sinus thrombosis, $6.67 \%$ had right transverse Sinus thrombosis, right jugular vein $4.4 \%$ had and $8.89 \%$ had left Jugular vein thrombosis.

Table (2): Distribution of different lesions detected by MRV examination.

\begin{tabular}{|l|c|c|}
\hline \multicolumn{1}{|c|}{ Lesion } & Number & Percentage (\%) \\
\hline Left Transverse Sinus thrombosis & 5 & $11.1 \%$ \\
\hline Right Transverse Sinus thrombosis & 3 & $6.67 \%$ \\
\hline Right jugular vein thrombosis & 2 & $4.4 \%$ \\
\hline Left Jugular Vein thrombosis & 4 & $8.89 \%$ \\
\hline
\end{tabular}

By MRA, $11.1 \%$ of patients had scattered atherosclerosis, $8.89 \%$ had right vertebral A. occlusion, $6.67 \%$ had left vertebral A. occlusion, $4.4 \%$ had right ophthalmic A. occlusion and $2.22 \%$ had basilar A. occlusion.

Table (3): Distribution of different lesions detected by MRA examination

\begin{tabular}{|l|c|c|}
\hline \multicolumn{1}{|c|}{ Lesion } & Number & Percentage (\%) \\
\hline Scattered Atherosclerosis & 5 & $11.1 \%$ \\
\hline Right Vertebral A. occlusion & 4 & $8.89 \%$ \\
\hline Left vertebral A. occlusion & 3 & $6.67 \%$ \\
\hline Right Ophthalmic A. occlusion & 2 & $4.4 \%$ \\
\hline Basilar A. occlusion & 1 & $2.22 \%$ \\
\hline
\end{tabular}

Among the study population, $36.7 \%$ of them showed hemorrhage, only $26.7 \%$ were seen by conventional MRI, and the rest of the cases are diagnosed by SWAN. Also, about $16.7 \%$ of patients were diagnosed with scattered microbleeds by SWAN and didn't visualize by conventional MRI.

Table (4) shows the value of adding SWAN to cMRI and in the diagnosis of chronic hemorrhage and microbleeds by percentage $(\%)$

\begin{tabular}{|l|c|c|c|c|}
\hline & \multicolumn{2}{|c|}{ Conventional MRI } & \multicolumn{2}{c|}{ SWAN } \\
\hline Lesion & Number & Percentage (\%) & Number & Percentage (\%) \\
\hline $\begin{array}{l}\text { Hemorrhage } \\
\text { (Rt parietal, Lt frontal, BG) }\end{array}$ & 8 & $26.7 \%$ & 11 & $36.7 \%$ \\
\hline Scattered Microbleeds & - & - & 5 & $16.7 \%$ \\
\hline
\end{tabular}

Table (5): Sensitivity, specificity, PVP, and PVN of SWAN with ADC value to detect brain hemorrhage

\begin{tabular}{|l|c|c|c|c|c|c|c|}
\hline Test & Cut off & Sensitivity & Specificity & ${ }^{\mathbf{a}} \mathbf{P V P}$ & ${ }^{\mathbf{b}} \mathbf{P V N}$ & Accuracy & ${ }^{\mathbf{c}} \mathbf{C I}$ \\
\hline ADC & $\geq 1000$ & $100 \%$ & $88.8 \%$ & $80 \%$ & $100 \%$ & $92.4 \%$ & $0.79-1.0$ \\
\hline
\end{tabular}

${ }^{\text {a }}$ Predictive value for positive $\quad{ }^{\mathbf{b}}$ Predictive value for Negative $\quad{ }^{\mathbf{c}}$ Confidence Interval

Table (6): Sensitivity, specificity, PVP, and PVN of DWI with ADC value to detect brain infarction

\begin{tabular}{|c|c|c|c|c|c|c|c|}
\hline Test & Cut off & Sensitivity & Specificity & ${ }^{\mathbf{a}}$ PVP & ${ }^{\mathbf{b}}$ PVN & Accuracy & ${ }^{\mathbf{c}} \mathbf{C I}$ \\
\hline ADC & $\leq 678$ & $100 \%$ & $100 \%$ & $100 \%$ & $100 \%$ & $100 \%$ & $0.93-1.0$ \\
\hline
\end{tabular}

$\begin{array}{lll}{ }^{\mathbf{a}} \text { Predictive value for positive } \quad{ }^{\mathbf{b}} \text { Predictive value for Negative } & { }^{\mathbf{c}} \text { Confidence Interval }\end{array}$ 

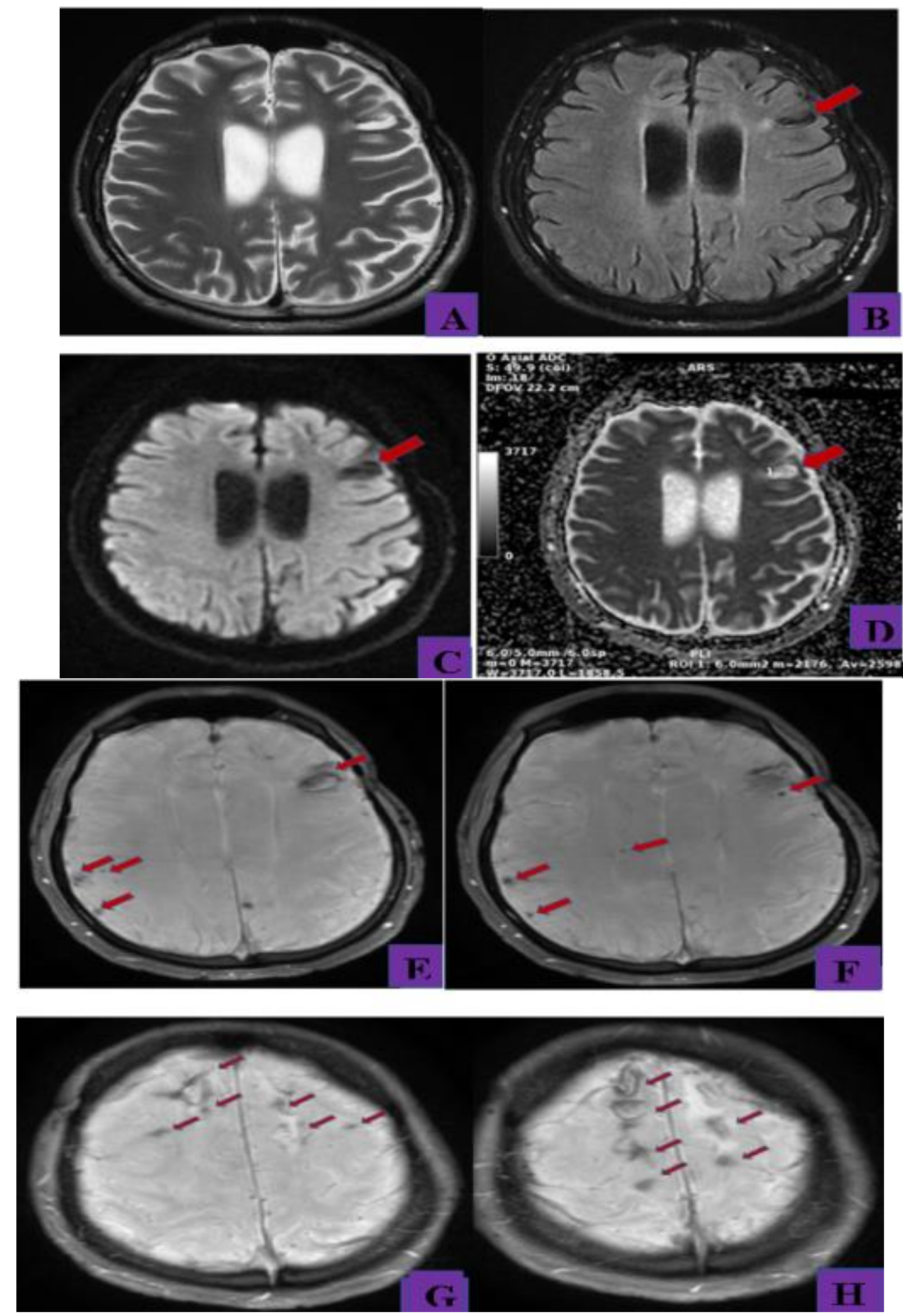

Figure (1): Case 1, 21-years-old male patient with ESRD on regular HD since 3-years presented by DCL and fits. Axial T2WI (image A) shows a high SI lesion at the left anterior parietal region with symmetrical dilatation of the lateral ventricle. Axial T2FLAIR (image B) shows a low signal intensity lesion at the left anterior parietal region with symmetrical dilatation of the lateral ventricle. Axial DWI (image C): the same lesion on T2FLAIR shows low SI. Axial ADC (image D): the same lesion shows high SI and ADC value: $2598 \times 10^{-6} / \mathrm{mm}^{2}$ low and correlative with hemorrhage. Axial SWAN (image E-H) shows multiple low signal intensity lesions scattered within the white matter. Scattered bleeding and microbleeds with brain atrophic changes and accelerated brain aging. 


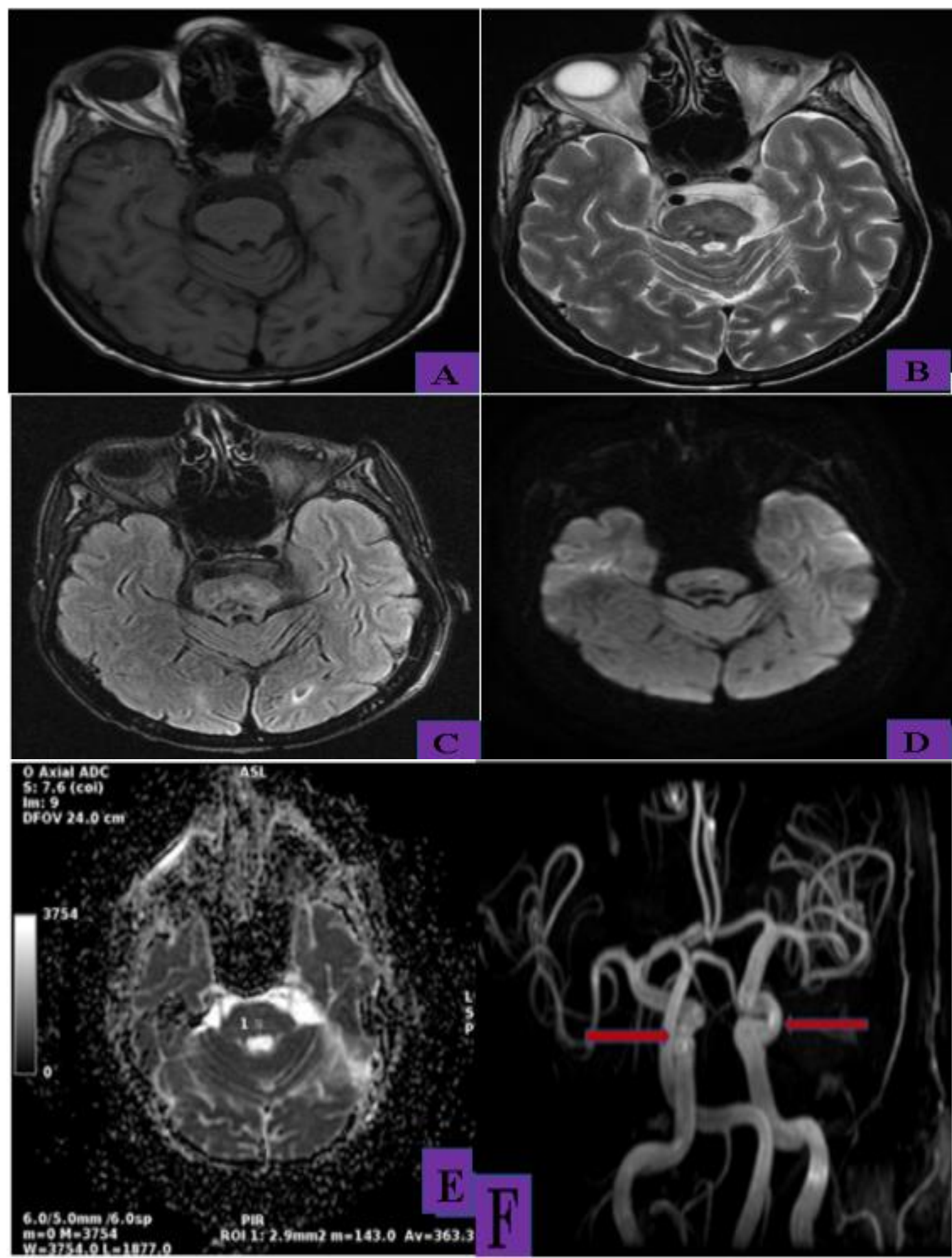

Figure (2): Case 2, 32-years-old Male patient with ESRD on regular HD since 6-months presented by dysarthria, bulbar symptoms, and imbalance. Axial T1WI (image A) shows pontine foci faint low SI. Axial T2WI (image B): shows pontine lesions of mixed-signal intensities, atrophic left orbit (phthisis bulbi) is seen. Axial T2FLAIR (image C) shows pontine lesions of mixed-signal intensities and the appearance of trident sign (omega sign). Axial DWI (image D) shows low signal intensities at pons. Axial ADC (image E) shows low signal intensities at pons and ADC value 363.3 $\mathrm{x} 10^{-6} / \mathrm{mm}^{2}$, low and related to old infarct measurement. MRA (image F) shows diffuse atherosclerosis. Pontine myelinosis (osmotic demyelination syndrome) with atherosclerosis and small artery disease, brain atrophy. 

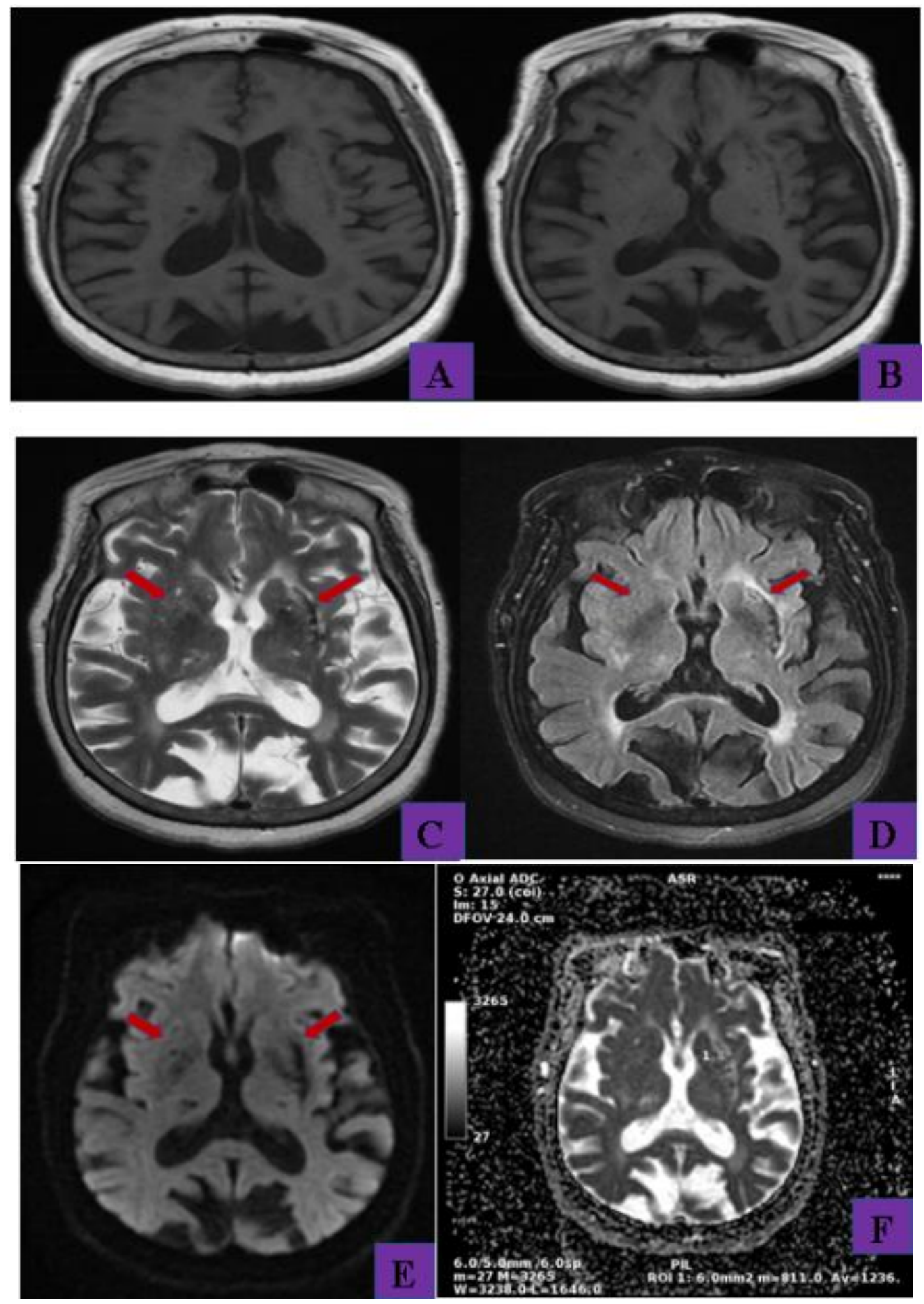

Figure (3): Case 3, 37-years-old-female patient with ESRD on regular HD since 4-years came to the out-patient clinic drowsy and suffering from paresthesia. Axial T1WI (image A\&B) shows few bilateral low SI BG lesions with dilated lateral ventricle. Axial T2WI (image C) shows bilateral BG lesions of high SI. Axial T2FLAIR (image D) hyperintense lesions at the area of basal ganglia with hyperintense rim surrounding bilateral putamina (red arrow). Axial DWI (image E) shows minute hypointense lesions at the basal ganglia region. Axial ADC (Image F) shows minute hyperintense lesions at basal ganglia region and ADC value (ROI1) $1236 \times 10^{-6} / \mathrm{mm}^{2}$, reduced. Lentiform fork sign with brain atrophy. 


\section{DISCUSSION}

Our study was conducted on 45 patients with ESRD. Their age ranges from 20-40 years, they were 18 female (40\%) and 27 males (60\%). All patients underwent conventional MRI, DWI with ADC, MRA, MRV, and SWAN.

Cerebral hemorrhage, infarction, atherosclerotic changes, and vascular occlusion (either arterial or venous) are the most common vascular brain insult seen in these patients. (31\%) of study participants by Gao et al. ${ }^{(7)}$ are recorded with vertebra-basilar occlusion and this is consistent with our study that found $33.28 \%$ of patients diagnosed by MRA with arterial occlusion and atherosclerosis.

In our study, we found that $(31 \%)$ of patients showed cerebral sinus venous thrombosis on MRV distributed along with both transverse sinuses and both jugular veins and this is consistent with El Damarawy et al. ${ }^{(8)}$ who diagnosed $(73.3 \%)$ of cases with sinus venous thrombosis of transverse sinuses and jugular veins using MRV.

Our findings showed that intracerebral hemorrhage was represented in (36.7\%) by conventional MRI and best seen by SWAN and this is consistent with Atluri et al. (9) who found that hemorrhage is an important neurological finding in those patients and it represents $(5 \%)$ of all examined patients and mentioned that SWAN is the most sensitive sequence to detect the intracerebral hemorrhage, also we found a statistically high sensitivity $(100 \%)$ and specificity $(88.8 \%)$ of SWAN of our cases which can detect microbleeds that may not appear with the conventional MRI and this is consistent with Zhang $\boldsymbol{e t}$ al. ${ }^{(10)}$ who found that the value of SWAN is higher in cerebral microbleeds diagnosis with more details than conventional MRI with significant $P$ value $(<0.05)$ and this helps in a better prognosis and early treating those patients. Also in comparison with ADC value measurements of the hemorrhage, we found our cases of chronic hemorrhage with cut off $\operatorname{ADC}$ value $(\geq 1000$ $\mathrm{x} 10^{-6} \mathrm{~mm}^{2} / \mathrm{s}$ ) and this is consistent with Kang et al. ${ }^{(11)}$ who found that the ratio of ADC value of the chronic stage of hemorrhage (2560).

Our study had different limitations as; 1-Small sample size. 2-Few patients couldn't withstand or tolerate the study duration. 3- Lake of a large number of previous researches discussing cerebral changes due to end-stage renal disease.

\section{CONCLUSION}

ESRD patients have many central neurological affections that are seen in MRI examination and not manifested clinically, so we recommend patients with ESRD patients on regular HD to be followed by MRI brain for proper management and to reduce or prevent morbidity and disability.

Financial support and sponsorship: Nil.

\section{Conflict of interest: Nil.}

References

1. Kang E, Jeon S, Choi S (2012): Uremic Encephalopathy with Atypical Magnetic Resonance Features on Diffusion-Weighted Images. Korean Journal of Radiology, 13:808-811.

2. Brouns R, De Deyn $P$ (2004): Neurological complications in renal failure: a review. Clin Neurol Neurosurg., 107(1):1-16

3. Fazel R, Krumholz H, Wang Y et al. (2009): Exposure to low-dose ionizing radiation from medical imaging procedures. N Engl J Med., 361(9):849-857.

4. Srivastava A, Mehrotra G, Bhargava S et al. (2008): Studies on the time course of the apparent diffusion coefficient and signal intensities on T2- and diffusionweighted MR Imaging in acute cerebral ischemic stroke. J Med Phys., 33:162-170.

5. Boulevard J, Brook O (2018): Magnetic Resonance, Functional (fMRI) - Brain, Radiological Society of North America, 18: 1-8.

6. Han B, Kim J, Kim B et al. (2015): Diffusion Tensor Imaging Predicts Motor Functional Outcome after Acute Hypertensive Intracerebral Hemorrhage. Journal of Cell Science \& Therapy, 6: 2-8.

7. Gao T, Yu W, Liu C (2014): Mechanisms of ischemic stroke in patients with intracranial atherosclerosis: A high-resolution magnetic resonance imaging study. Experimental and Therapeutic Medicine, 7: 1415-1419.

8. El Damarawy E, El-Nekiedy A, Fathi A et al. (2012): Role of magnetic resonance venography in the evaluation of cerebral veins and sinuses occlusion. Alexandria Journal of Medicine, 48: 29-34.

9. Atluri M, Borra S, Jakkamsetti S et al. (2016): Role of MRI in Detection of Neurological complications in Patients with Chronic Kidney Disease. Journal of Dental and Medical Sciences, 15: 17-25.

10. Zhang D, Xu L, Ma A et al. (2017): Value of conventional MRI and susceptibility-weighted imaging in diagnosis of cerebral microbleeds. Biomedical Research, 28(20): 8905-8908.

11. Kang B, Na the D, Ryoo J et al. (2001): DiffusionWeighted MR Imaging of Intracerebral Hemorrhage. Korean J Radiol., 2:183-191. 